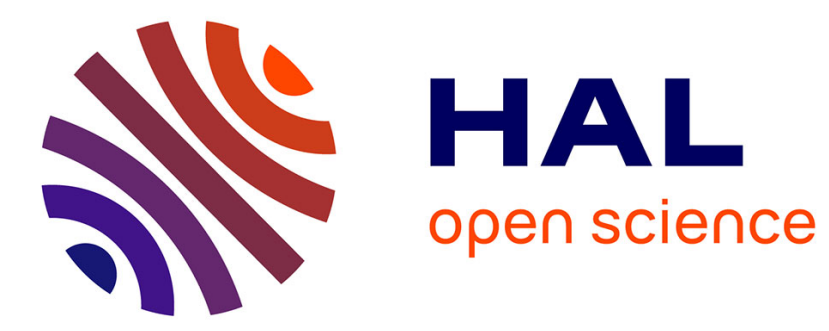

\title{
Le Désordre nouveau : Violence sociale et changement politique en Indonésie
}

\author{
Romain Bertrand
}

\section{To cite this version:}

Romain Bertrand. Le Désordre nouveau: Violence sociale et changement politique en Indonésie. Critique Internationale, 2000, 8, pp.28-35. 10.3406/criti.2000.1436 . hal-01011337

\section{HAL Id: hal-01011337 \\ https://hal-sciencespo.archives-ouvertes.fr/hal-01011337}

Submitted on 23 Jun 2014

HAL is a multi-disciplinary open access archive for the deposit and dissemination of scientific research documents, whether they are published or not. The documents may come from teaching and research institutions in France or abroad, or from public or private research centers.
L'archive ouverte pluridisciplinaire HAL, est destinée au dépôt et à la diffusion de documents scientifiques de niveau recherche, publiés ou non, émanant des établissements d'enseignement et de recherche français ou étrangers, des laboratoires publics ou privés.

\section{(ㅇ)(1) $\$$}

Distributed under a Creative Commons Attribution - NonCommercial - NoDerivatives| 4.0 


\title{
Le Désordre
}

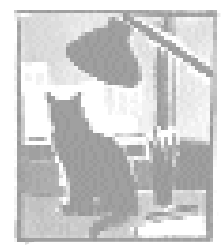

\section{nouveau}

\author{
Violence sociale et changement \\ politique en Indonésie
}

par Romain Bertrand

$\ll$

démocratie du monde », affirmait Megawati

Sukarnoputri, dirigeante du Parti démocra-

tique indonésien-Combat (PDI-P), après sa victoire aux élections du 7 juin 1999 : optimisme partagé par les représentants de plusieurs grandes agences internationales spécialisées dans la gestion du « passage à la démocratie ». Après avoir longuement conseillé la Commission électorale sur le choix d'un mode de scrutin approprié à la première consultation libre depuis plus de quarante ans, le National Democratic Institute, à l'instar de la plupart des équipes de « surveillance » des lieux de vote, se félicitait du calme qui avait régné le jour du vote comme après l'annonce des résultats. Enfin, la majorité des grandes chancelleries occidentales, et en premier lieu les États-Unis qui avaient opté pour une « conditionnalité » très ferme subordonnant l'attribution d'aides financières internationales à la tenue de ces élections libres, avaient toutes les raisons de se réjouir. La Reformasi (réforme) était bien le prélude à la demokrasi.

Cri de guerre des mouvements d'étudiants depuis le milieu des années quatrevingt, le terme de Reformasi désignait à l'origine un programme de libéralisation de la vie politique articulé autour de trois revendications majeures : extension des prérogatives du Parlement et amendement de la Constitution de 1945 pour mettre fin à la dérive présidentialiste des institutions, « retour aux casernes » de l'armée de terre, fin de la collusion entre grands industriels, militaires de haut rang et hommes politiques. La Reformasi devait donc marquer la sortie du système autoritaire mis en place par le général Suharto en 1965-1966 et connu sous l'appellation d'Ordre nouveau (Orde Baru) : un système gangrené par la violence arbitraire, la corruption et le népotisme.

Le gouvernement formé par B.J. Habibie - successeur du président Suharto contraint à la démission le 21 mai 1998 - baptisé, dans une tentative classique de récupération du slogan initial des meneurs étudiants, « gouvernement de réforme et de développement », n'eut d'autre choix, devant l'insistance des diplomates étrangers et la menace persistante de nouvelles violences de rue, que d'engager un 
processus de refonte du système politique national. L'octroi de la liberté de la presse et la promesse de renoncer à l'usage des moyens de terreur créés par l'Ordre nouveau, en particulier en séparant forces de police et forces armées, complétait un dispositif législatif censé favoriser la renaissance du débat public et l'expression non violente des demandes sociales. L'élection à la tête de l'État, le 20 octobre 1999, d'un leader musulman modéré, Abdurrahman Wahid, annonçait même une victoire au sommet contre les « forces réactionnaires ». Peu à peu, on voyait se ranger aux côtés du Président une faction réformiste de l'armée de terre emmenée par le lieutenant-colonel Agus Wirahadikusumah. On s'empressa de prophétiser la soumission du pouvoir militaire au pouvoir civil.

\section{L'armée aux aguets, les partis aux abois : la faiblesse de la « société civile »}

La réalité allait démentir par deux fois l'optimisme de cette approche « transitologique ». En premier lieu, on pouvait douter de l'existence d'un « pouvoir civil » autonome et légitime. Bien que quarante-huit partis se soient affrontés lors des élections du 7 juin 1999, peu d'entre eux possèdent des liens réels avec leur électorat potentiel. Les discours les plus populistes restent viscéralement élitistes et la plupart des partis se sont très rapidement délités après le scrutin. Dans certains cas, leurs états-majors, minés par un factionnalisme qu'avivait la perspective du pouvoir, s'entre-tuèrent. Le PDI-P de Megawati Sukarnoputri, clivé entre une coterie de notables opportunistes et des mouvements de jeunesse peu enclins aux concessions, illustre assez bien l'une des trajectoires d'affaissement. Dans d'autres cas, l'échec électoral a suffi à faire voler en éclats les alliances de circonstance nées d'apparentements de dernière minute entre petites formations.

Si des associations telles qu'Internews cherchent à développer un espace public indonésien via les ondes et le Web, les « relais sociaux » du débat d'idées font cruellement défaut. Les syndicats sont fragmentés, les clubs politiques peu nombreux et concentrés dans les grandes cités. La plupart des partis n'ont pas su prendre appui sur ces lieux populaires du politique que sont les petits restaurants de bord de route (warung) et les porches des mosquées. Abdurrahman Wahid n'est lui-même que le leader d'un parti arrivé en quatrième position lors du scrutin, et qui ne dispose que de cinquante et un sièges sur quatre cent soixante-deux à la Chambre basse. Les élections n'ont pas permis d'apaiser les tensions qui menacent la recomposition d'une communauté politique fondée sur l'universalité des droits. Le racisme anti-chinois demeure largement répandu, même si l'attribution du portefeuille de l'Économie à Kwik Gian Gie (PDI-P) a quelque peu rassuré la communauté sinoindonésienne. La très violente mobilisation anti-chrétienne de ces derniers mois à propos des Moluques montre que l'échec électoral de l'islam politique n'est aucunement le gage d'une accalmie religieuse dans l'archipel. Au contraire, les 
groupuscules islamiques écartés du pouvoir d'État tentent de faire pression sur ce dernier en recourant à la manifestation violente et à la menace de jihad. Sous la houlette de prédicateurs connus, des mouvements de jeunesse islamique comme le Front des défenseurs de l'islam (FPI) mènent des expéditions punitives dans les banlieues de Jakarta, brûlant les « lieux de perdition » (maisons de plaisir, salons de massage, discothèques, salles de jeu).

En second lieu, alors qu'on disait l'armée de terre à l'agonie, celle-ci a su affermir son emprise sur des territoires lucratifs comme l'Irian Jaya (partie indonésienne de la Nouvelle-Guinée) et Aceh (nord de Sumatra) ${ }^{1}$. Les ressources naturelles de ces territoires (minerais et hydrocarbures) sont affermées à de puissantes multinationales, qui n’hésitent pas à rémunérer des unités militaires pour protéger leurs installations et maintenir leur main-d'œuvre locale dans la docilité. Pendant la période d'occupation militaire d'Aceh (1988-1998), Mobil Oil, qui exploitait avec la société PT Arun le complexe industriel de Lhokseumawe, a mis à la disposition des Kopassus (commandos spéciaux de l'armée) certains de ses bâtiments, transformés en centres de torture ${ }^{2}$.

La tragédie timoraise a d'autre part fourni à l'armée l'occasion de s'aligner sur le nationalisme séculariste de Megawati Sukarnoputri, et donc de réaffirmer son rôle de gardienne de l'intégrité de la nation. Après la sécession du Timor oriental, les militaires ont pu dire à des foules échauffées par l'arrogance australienne : «Plus jamais ça !». La création des nouveaux commandements territoriaux, la reformulation des doctrines de sécurité intérieure, l'augmentation substantielle de son budget et la captation du ministère des Transports et de celui des Mines ont permis à l'armée de terre de préserver sa mainmise sur le business mercenaire des «zones-frontière ». Trafics de pierres et de métaux précieux ou de drogues se poursuivent dans les jungles de Kalimantan ou d'Irian Jaya. Pas une semaine ne s'écoule sans que des officiers, souvent proches des unités les plus déconsidérées (comme les Kopassus), ne soient impliqués dans des affaires de revente de stupéfiants, de contrebande de biens de consommation coûteux ou d'extorsion de fonds. Même s'ils ont perdu plus de la moitié de leurs sièges réservés au Parlement, et même si se resserre l'étau de la Commission nationale des droits de l'homme autour des généraux les plus compromis au Timor oriental, les militaires ont réussi à conserver un rôle central dans la vie économique et politique, en particulier au niveau local. La reprise des exactions à Aceh, depuis le mois de février, l'illustre assez.

Sans vouloir rouvrir ici le procès de la « transitologie » comme science du changement politique contemporain ${ }^{3}$, on peut s'interroger sur ce qu'elle laisse systématiquement de côté, notamment les raisons culturelles de l'action politique. Il n'a ainsi été question, dans l'interprétation du mouvement indonésien de Reformasi, que du jeu des leaders. Quand l'analyse se tournait vers les campagnes de Java, les 
docks de Surabaya ou les collines d'Aceh (à Sumatra), ce n'était, le plus souvent, que pour mieux souligner le divorce entre une Indonésie « moderne », parlant le langage internationalement gratifiant des parpol (partis politiques) et des scrutins jurdil (de jujur et adil : traduction littérale de free and fair) et une Indonésie des villages, qui croit aux esprits et ne comprend rien à la demokrasi. Indonésie du building de verre et d'acier ouverte aux vents du large et Indonésie du desa, du hameau engoncé dans son archaïsme et une peur irraisonnée de la nouveauté : que vaut cette dichotomie?

En termes purement topographiques, sa valeur heuristique est des plus médiocres. Il suffit, à Jakarta, de quitter le salon aseptisé d'un grand hôtel abritant un important séminaire sur la demokrasi, puis d'emprunter une ruelle latérale, pour découvrir l'univers bigarré des échoppes, croiser les visages marqués des prostituées et des enfants des rues qui attendent la tombée du soir pour « traquer le sou », et s'émerveiller de la prodigieuse inventivité $\mathrm{du}$ « petit peuple » en matière de survie. Surabaya et ses bordels ne sont qu'à une heure et demie de vol de Jakarta, le Timor oriental et ses charniers à deux heures et demie, Aceh et ses vallées ensanglantées à trois heures. L'Indonésie des gratte-ciel et celle des rizières, quoi qu'ait pu en dire un régime autoritaire qui concevait souvent la modernisasi comme une partie de chasse aux croyances rurales, ne forment pas deux univers étrangers l'un à l'autre. Il existe entre eux un réseau dense de «points de connexion » matériels et imaginaires : routes, canaux hertziens, ferries, trains, journaux, courrier électronique, romans.

Pour ressaisir l'économie morale de la Reformasi - c'est-à-dire pour mettre au jour les rêves, les fantasmes, les idées préconçues sur le rôle du notable ou les devoirs de l'État qui ont informé les discours et les comportements politiques du plus grand nombre au fil des tragiques événements des deux années passées -, c'est l'architecture complexe des relations de pouvoir qu'il est indispensable de garder à l'esprit. Par le biais d'institutions à finalité surtout disciplinaire - écoles, prisons, casernes, dispensaires publics -, l'Ordre nouveau a cherché à pénétrer jusqu'au cœur des villages pour y inculquer les rudiments d'une existence « ordonnée » (diatur $)^{4}$. Ce faisant, il n'a pas fait que bâtir des ponts ou des bureaux de poste. Il a aussi imprégné les relations sociales d'un imaginaire spécifique du pouvoir, qui perdure après le renversement du tyran. Cet imaginaire n'est pas seulement un outil d'oppression. C'est un champ sémantique ouvert, un régime d'énonciation du fait de domination englobant simultanément le dit des dominés et celui des dominants, le murmure des idéologies et le cri utopique. Comme alternative à la lecture linéaire du «passage à la démocratie », une escapade dans les sous-bois de l'entendement politique populaire prenant appui sur l'élucidation de cet imaginaire permet de dépasser la coupure artificielle qu'établit la vulgate transitologique entre Indonésie des villes et Indonésie des champs, « modernité » et « tradition ». 


\section{Quand les villageois tuent les fous qui tuent les sorciers: les ressorts moraux de la violence populaire}

À l'automne 1998, tandis que se créaient à Jakarta plus d'une centaine de formations politiques aux motivations ambiguës, la presse locale et nationale publiait d'étranges récits. D'après le compte rendu de la commission d'enquête créé par l'association musulmane Nahdlatul Ulama, près de deux cent cinquante sorciers (dukun santet, tukang santet) auraient été assassinés, entre août et décembre 1998, aux alentours de la ville de Banyuwangi (Java-Est) 5 . La rumeur attribuait ces meurtres inexpliqués à de petits commandos d'hommes cagoulés, bientôt baptisés Ninjas. Les journaux ne tardèrent pas à faire passer ces incidents sanglants de la rubrique des faits divers aux pages d'analyse politique ${ }^{6}$. Curieusement, en effet, les Ninjas ne semblaient pas mus par l'appât du gain, mais par une incompréhensible cruauté : ils agissaient d'une manière « sadique », mais « soigneusement »7. Pénétrant par effraction, à la nuit tombée, dans les demeures de leurs victimes, ils les bâillonnaient puis les emmenaient dans une voiture ou une fourgonnette jusqu'au lieu de leur « abattage » (pembantaian, de bantai, animal de boucherie), où elles subissaient d'effroyables tortures avant d'être exécutées et leurs cadavres « jetés » aux abords des villages ou abandonnés à la lisière des forêts.

Guettées comme des « proies», les victimes étaient subitement arrachées à la paix de leur foyer pour gagner des lieux inconnus ${ }^{8}$. Cette image, indéfiniment répétée, du trajet depuis l'espace clos et paisible (diatur, aman) du desa (village) jusqu'aux confins de la sylve sauvage (butan, la forêt, refuge des démons et des brigands) renvoie à l'idéal autoritaire d'une nation de petites unités sociales autarciques, greffées sur les rites de la capitale mais imperméables au conflit politique. Or c'est cet imaginaire, bribes de propagande de l'Ordre nouveau, qui devient, pour les villageois, justification et moyen de l'action politique. Car la question qui est alors sur toutes les lèvres est, bien entendu, celle de l'identité du commanditaire de ces « meurtres mystérieux » qui évoquent d'horribles précédents9. Qui ordonne le pembantaian, le massacre sanglant des sorciers ? S'agit-il d'une faction conservatrice de l'armée de terre souhaitant faire obstacle à la poursuite de la Reformasi, comme l'affirme « Gus Dur » (Abdurrahman Wahid) ${ }^{10}$, ou bien, comme le clame l'armée à cor et à cri, d'une résurgence de la menace communiste due au « désir de revanche » des descendants des victimes du «drame » de 1965-1966 ${ }^{11}$ ? La panique se répand dans les villages de la région. On organise des rondes, on crée des milices armées jusqu'aux dents, on invite chacun à se barricader pour la nuit ${ }^{12}$. Dès le mois d'octobre, dans ce climat d'extrême tension, des groupes d'autodéfense improvisés décident de « faire justice eux-mêmes ». En quelques semaines, les lynchages de suspects se multiplient. Tout individu étranger au village se voit sommé par des foules haineuses de décliner son identité (asal-usul, sa provenance 
et sa généalogie) sous peine d'être rossé. Pour se prémunir contre les attaques des Ninjas, les villageois immolent des sorcières présumées. L'une d'elles est brûlée vive après avoir été pendue à un arbre ${ }^{13}$.

Le climat d'hystérie était entretenu par les rumeurs les plus folles sur l'arrivée imminente de camions de Ninjas. Aux environs de Malang, le 19 octobre, le corps mutilé d'un « inconnu » fut exhibé à l'arrière d'une moto lors d'une macabre procession à travers la ville, sa tête empalée sur une pique de bambou. Le funèbre convoi se rendit au poste de police de Kenpajen, à 75 kilomètres de là. L'inconnu, accusé d'appartenir à un groupe de Ninjas, avait été mis à mort par une foule d'une cinquantaine de personnes ${ }^{14}$. Dans un autre cas de lynchage à Malang, la victime était un homme âgé de vingt-sept ans. Soupçonné d'être un Ninja, Supriono était connu pour être, depuis l'enfance, un simple d'esprit (kurang waras). Il fut surpris « alors qu'il sommeillait près d'un oratoire, à Kebalen, [et il tenta] de s'enfuir tandis qu'on l'interrogeait sur ses origines ${ }^{15}$.

La chasse aux inconnus se transforme ainsi brusquement en chasse aux fous (orang gila, ou orgil). Parce qu'il ne peut énoncer clairement son «identité sociale » - et aussi parce qu'il appartient à cette frange errante de la population qu'on nomme les gelandangan (vagabonds) -, le dément connaît le même sort tragique que le migrant. Roué de coups, il est abandonné, ensanglanté, aux limites du village. Cette surenchère populaire de terreur atteint des proportions telles que la police locale décide de «mettre en sécurité » les fous et de « prendre soin »d'eux : elle en arrête cent quarante au cours de rafles dans les faubourgs miséreux, puis les parque dans des établissements d'une insalubrité telle que même les policiers chargés de leur internement en éprouvent du dégoût ${ }^{16}$. La foule prend parfois d'assaut les postes de police, lapide des détectives, ou met à sac des casernes, pensant que l'armée est de mèche avec les Ninjas ${ }^{17}$. Autrement dit, les villages de l'est de Java se purgent par la violence de tout ce que les habitants perçoivent comme un en-dehors hostile de leur quotidien. Policiers, soldats, vagabonds, fous, magiciens, inconnus de passage... On voit ici se dessiner la figure de l'Extérieur. Ceux qui sont mis à mort avec une brutalité inouie sont les intercesseurs avec les forces qui échappent au contrôle moral communautaire (l'État, les esprits, les itinérants de toutes sortes).

Il s'agit là à l'évidence d'un type de faits que les observateurs « transitologues » de la situation politique indonésienne relèguent dans la case miscellaneous de leur grille de lecture : événements incongrus qui ne peuvent relever que de la folie des foules, ou bien faits apolitiques qui ne méritent pas de retenir l'attention de l'analyste sérieux du « passage à la démocratie ». Or l'affaire des Ninjas de Banyuwangi a pris place exactement au moment de la renaissance du débat d'idées en Indonésie, et elle a passionné le pays pendant plusieurs mois. On peut donc faire l'hypothèse que la manière dont cette série de meurtres tragiques a été interprétée participait à la formation de l'espace public indonésien. Car de quoi était-il question, dans les 
articles consacrés aux Ninjas et aux lynchages d'étrangers dans les villages, sinon du dilemme de la territorialisation des allégeances et de la redéfinition de l'identité citoyenne? Ceux qui ont subi le courroux des villageois étaient ceux-là mêmes dont le statut politique posait problème. Il fallait tout d'abord donner sens au passé autoritaire en se prononçant sur la réintégration dans la communauté nationale de ceux qui avaient subi la violence de l'Ordre nouveau, comme les « communistes $\gg$. Il fallait ensuite interroger une définition de la citoyenneté qui subordonnait l'octroi de droits politiques à la possession d'une identité « stable », caractérisée par l'appartenance à un lieu et à des catégories (« ethniques », professionnelles) reconnues car énoncées par l'État. Le fou et le migrant échappaient à cette conception sédentaire de la citoyenneté : c'est pour cela qu'ils devinrent les cibles du courroux populaire. Cela prouve que ce courroux était encore tributaire de l'idéologie de l'Ordre nouveau, qui avait souhaité sur le tard composer avec le rigorisme islamique pour se ménager des soutiens.

Le massacre des sorciers et l'enfermement des fous n'ont d'ailleurs pas été les seuls faits attestant l'appropriation populaire des problèmes centraux de la sortie du « régime Suharto ». Bon nombre de lieux de plaisir et de vice ont été détruits un peu partout à travers l'archipel, en 1998-1999, à l'appel de mouvements de jeunesse islamique engagés dans une véritable « croisade morale ». Faut-il à nouveau ranger ces faits dans la catégorie des « incidents de parcours du passage à la démocratie », les inscrire au passif du changement politique, comme certains ont souhaité le faire du drame du Timor oriental ? Faut-il les occulter au nom d'un paradigme qui réduit l'Indonésie aux salons de l'hôtel Indonesia, ou encore condamner les insurrections paysannes et les grèves des dockers au prétexte qu'elles ne sont que de l'anarkisme préjudiciable au bon déroulement de « la transition » ? Ou bien faut-il au contraire interroger à travers eux un canevas d'attentes populaires qui ne correspondent que très imparfaitement aux centres d'intérêt des nouveaux partis politiques ? Ce que la haine à l'encontre des migrants ou des prostituées révèle, c'est une vision de l'avenir politique désirable comme entreprise de moralisation de la vie sociale, et aussi le refus d'une litanie de promesses et de décrets qui ne sont que rarement en prise sur les soucis et les souhaits du plus grand nombre.

Mais ce que dévoile en sus l'étude de ce commentaire social de la Reformasi, c'est l'incapacité présente des communautés villageoises de Java à donner un sens au changement politique autrement qu'en puisant dans le stock des mythes de gouvernement de l'Ordre nouveau : malgré de réels progrès en termes de retour des libertés publiques, le débat sur la citoyenneté et l'attribution des droits politiques reste prisonnier des fantasmes collectifs hérités du passé autoritaire. La peur du migrant, la méfiance haineuse à l'égard du vagabond ou de la prostituée doivent beaucoup à l'« hygiénisme social » de l'Ordre nouveau. Bref, il n'y a pas de solution de continuité entre l'économie morale de l'autoritarisme et celle de la Reformasi. 
Mais que les mots et les dilemmes du pouvoir survivent au naufrage des régimes ne signifie pas que se soit tarie l'imagination politique du « petit peuple ». C'est, au contraire, en faisant usage de ce même matériau imaginaire que les villageois de l'est de Java et les habitants des bidonvilles de Jakarta peuvent se mouvoir hors du discours creux et superficiellement progressiste des hérauts autoproclamés de la Reformasi. À charge pour eux, cependant, de réinventer, peut-être contre l'État et les partis, le refus de la violence.

1. La réorganisation interne de l'armée de terre a commencé dès la fin 1998. Mais l'éviction du général Wiranto, souhaitée tant par les États-Unis que par Abdurrahman Wahid, a accéléré le processus et rééquilibré les factions en présence. Une vague de mutations, en novembre 1999, a porté à la tête d'importantes unités des partisans d'une « professionnalisation » de l'activité militaire, tandis que, sous la direction de l'amiral A.S. Widodo, des arbitrages budgétaires cruciaux ont été faits au profit de l'armée de l'air et de la marine, soutiens de Megawati Sukarnoputri.

2. «Indonesia : What did Mobil Oil know ?», Business Week, 28 décembre 1998.

3. Voir notamment Guy Hermet, « Le charme trompeur des théories : un état des travaux », dans C. Jaffrelot (dir.), Démocraties d'ailleurs, Paris, Karthala, 2000, pp. 315-342.

4. Le mythe du desa autarcique qui remonte à Sir T.S. Raffles et à l'imaginaire orientaliste néerlandais de la Dorpsrepubliek a été amplement repris à son compte par l'Ordre nouveau. Voir en particulier Hans Antlöv (dir.), Exemplary Centre, Administrative Periphery : Rural Leadership and the New Order in Fava, Richmond, Curzon Press NIAS, 1995.

5. « East Java killings act of political terror says NU », Tempo, 14 décembre 1998.

6. «Teror santet : ABRI sampai menteri kabinet kena tuduh », Gatra 50/IV, 31 octobre 1998.

7. « Operasi Ninja, operasi intelijen », Gatra 50/IV, 31 octobre 1998.

8. «Isu Ninja dibantah», Fawa Pos, 13 novembre 1998.

9. Voir Justus van der Kroef, « Petrus : Patterns of prophylactic murder in Indonesia », Asian Survey, n² 25, 1985, pp. 745759, pour une étude de la vague d'assassinats de petits délinquants à Java en 1982-1983.

10. « Naga Hijau di mana-mana », Gatra 50/IV, 31 octobre 1998 ; « Isu santet seperti operasi Naga Hijau », Suara Merdeka, 18 octobre 1998, pour le détail des propos de Gus Dur.

11. « Pangdam v brawijaya mayjen TNI Djoko Subroto, serangkaian peristiwa di Jawa Timur, ditumpangi politik kelompok revolusioner », Kompas, 5 novembre 1998 ; « Peristiwa Jatim ditumpangi kelompok revolusioner », Kompas, 6 novembre 1998. Voir aussi les propos d'un membre du groupe parlementaire de l'ABRI, Yusuf Hasyim, dans « Ini perbuatan orang komunis », Tempo, 17 octobre 1998 et « Setan topeng di tapal kuda », Gatra 49/IV, 24 octobre 1998. En 1965-1966, l'armée, sous la direction de Suharto, avait organisé le massacre de plus de 600000 communistes présumés.

12. «Melacak pembunuh berantai banyuwangi », Gatra 47/IV, 10 octobre 1998 ; « Muncul dugaan aparat lindungi Ninja », Suara Merdeka, 6 novembre 1998 ; « Hadapi Ninja NU bentuk Pasukan siluman Putih », Fawa Pos, 26 octobre 1998 ; « NU Kirim Pasukan Anti-Ninja », Surabaya Post, 15 octobre 1998.

13. « The black death », The Sydney Morning Herald, 7 novembre 1998 ; « Warga mengamuk, satu tewas dibakar », Republika, 24 novembre 1998.

14. Des cas semblables sont mentionnés dans « Fears of sorcerers spur killings in Java », The New York Times, 20 octobre 1998.

15. «Tersangka Ninja ditebas kepalanya di Malang », Suara Pembaruan, 20 octobre 1998.

16. « Hari pertama operasi di Jateng. 200 orang gila dikumpulkan », Suara Pembaruan, 6 novembre 1998 ; « Polda Jateng amankan 200 orang sakit jiwa », Kompas, 5 novembre 1998 ; « Operasi menjaring gelandangan sakit jiwa di Bogor », Suara Pembaruan, 10 novembre 1998 ; « Saya dikatakan Ninja, apak nggak hebat. Ciaaattt ! , Fawa Pos, 13 novembre 1998.

17. «Soal kerusuhan massa di Semampir. Bukan Ninja tapi petugas yang lakukan penyelidikan tertutup », Surabaya Post, 15 octobre 1998 ; « Mob raids Surabaya police station over sorcerer », Suara Merdeka-Online, 18 octobre 1998 ; « Mapolres kuningan dirusak massa yang cari Ninja », Kompas, 4 novembre 1998. 